\title{
Design of Translator Glove for Deaf-Mute Alphabet
}

\author{
Carlos Pesqueira Fiel, Cesar Cota Castro, Victor \\ Velarde Arvizu \\ Departamento de Ingeniería Industrial-IME \\ Universidad de Sonora \\ Hermosillo, México \\ carlos_pesque@hotmail.com \\ [cesar.cotac][vicvea290991]@gmail.com
}

\author{
Nun Pitalúa-Díaz, Diego Seuret Jiménez, Ricardo \\ Rodríguez Carvajal \\ Departamento de Ingeniería Industrial-DIFUS. ITE \\ Universidad de Sonora \\ Hermosillo, México \\ [npitalua][ricardo]@industrial.uson.mx \\ diego@seuret.com
}

\begin{abstract}
This manuscript present the operation and construction of a glove that help deaf-mute communication by identifying the position of the fingers of the hand to translate the letters into words and send audio signals. Also we present an improve in the translator glove by adding a portable voice synthesizer and a microcontroller instead of using a computer or smartphone.
\end{abstract}

Keywords-electronic circuit; microcontroller; sensor systems

\section{INTRODUCTION}

Nowadays the world counts with similar projects that need the use of components that are not in a translator glove, these elements are smartphones and computers that are used for compilation of data and the presentation of text. This research has a microcontroller and speech synthesizer so that components make the translator glove portable and easy to use every day.

Today practice and fast communication is a necessity for daily performance, for it is believed in supporting people who do not have a way to communicate easily, such as the deaf.

The deaf-mute people based their communication on various types of sign languages, these are based from an alphabet of signs called "universal fingerspelling" (Fig. 1), which is the one used in this project, until languages based on signs.

The use of a support device in the communication for a deaf-mute person may give him/her a better quality of life to work in basic situations. World Health Organization [1] mentions:

In developing countries, children with hearing loss and deafness rarely receive any schooling. Adults with hearing loss also have a much higher unemployment rate. Among those who are employed, a higher percentage of people with hearing loss are in the lower grades of employment compared with the general workforce.

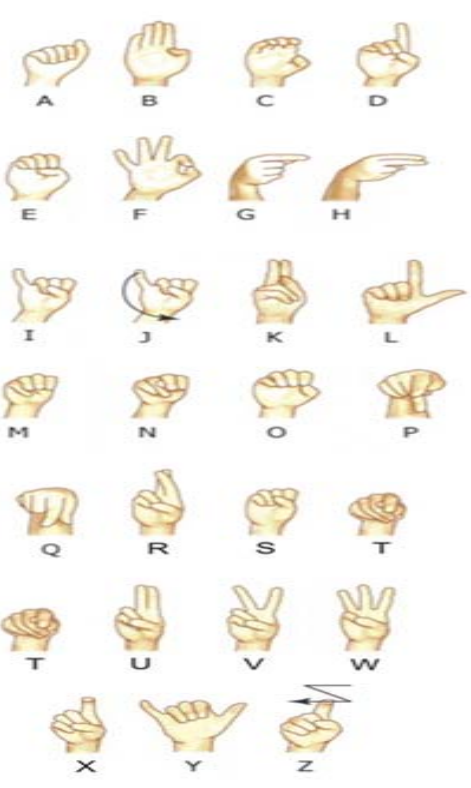

Fig. 1. Universal fingerspelling (pensadoresdelaurbe.wikispaces.com)

Over 5\% of the world's population - 360 million people - has disabling hearing loss (328 million adults and 32 million children). Disabling hearing loss refers to hearing loss greater than $40 \mathrm{~dB}$ in the better hearing ear in adults and a hearing loss greater than $30 \mathrm{~dB}$ in the better hearing ear in children.

The foundations and / or associations to support these people are based only on teaching sign language leaving aside the technology.

People with hearing loss can benefit from devices such as hearing aids, assistive devices and cochlear implants, and from captioning, sign language training, educational and social support [1].

One of the most important parts of the paper is translate the universal fingerspelling signs into audio signals.

\section{Translator GLOVE DESIGN}

This research consists in some resistive sensors and an accelerometer placed on a glove, which uses the technique 
employ as in [2-5] and detects the exact position of the fingers and the movements made by the hand.

Conducted a data acquisition from the sensors by $\mathrm{C}$ language programming to identify the alphabet letters formed with fingers. Taking these data, words are formed and will be converted to audio signals

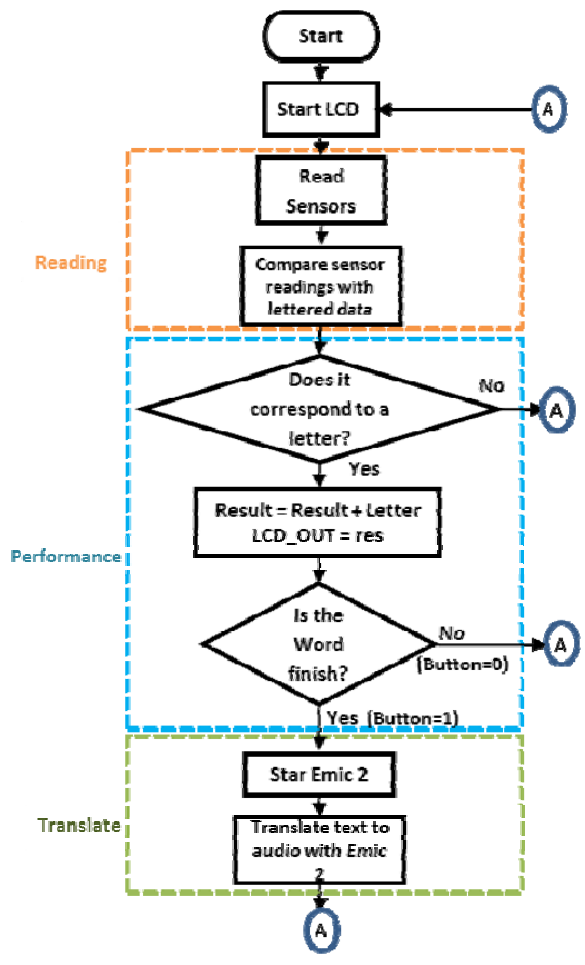

Fig. 2. Flowchart of main program

For a more thorough explanation the system can be divided into three stages (Fig. 2):

- Reading

- Performance

- Translation

\section{A. Reading.}

The components included in the reading step are:

- Bend Sensor. - This sensor is a variable resistor which increases resistance when it is bending and decreases otherwise (Fig. 3).

- Pressure sensor. - Consists of a variable resistor, this will vary its resistance depending on how much pressure is being applied to the sensing area. Harder the force, lower the resistance (Fig. 4).

- Accelerometer. Consists of recorder the variations of the position on 3 axes coordinate.

- Signal conditioning circuit. - This circuit is used to amplify the signal obtained from the variation of resistive sensors.

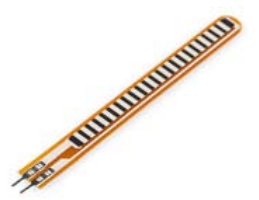

Fig. 3. Bend Sensor (sparkfun.com)

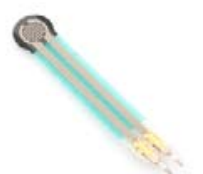

Fig. 4. Force sensitive resistor (sparkfun.com)

The signal conditioning circuit contains 10 bend sensors and 3 pressure sensors which provide us with a voltage variation to be bent or pushed either case (Fig. 5). This variation is acquired by a signal amplifier circuit in order to increase the variation given by the sensor and so to manage it more accuracy. This signal is sent to the analog-digital converter of PIC16F887 to be read by it, then it will compare with the information of the other sensors signals.

\section{A. Performance}

The components included in the processing stage are:

- $\quad$ Microcontroller PIC16F887 (microchip.com).

- Program.

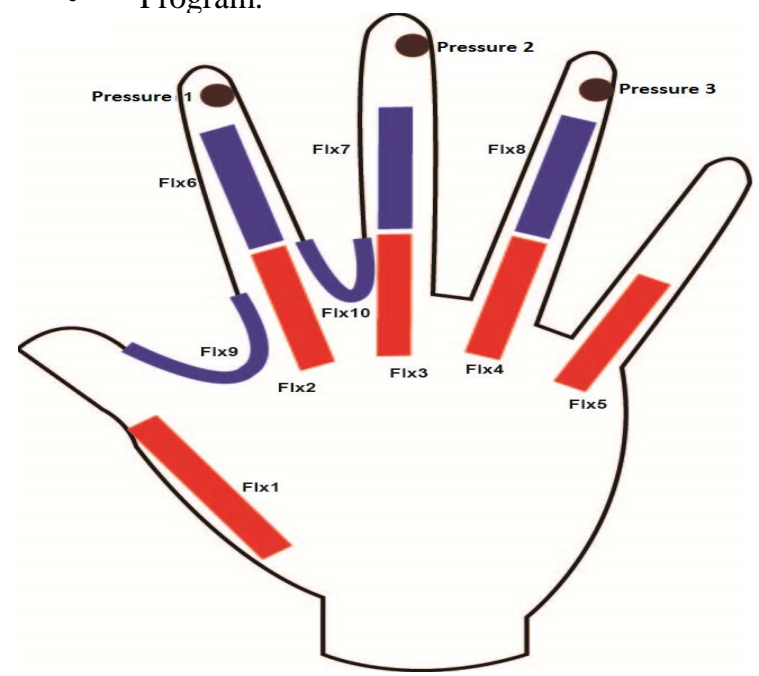

Fig. 5. Distribution of sensors ( $\mathrm{fl} \mathrm{x}=$ bend sensor)

Functioning at this stage:

When the sensor is not bent, the sensor is $0^{\circ}$ (Fig. 6), this sensor registration performed by the PIC16F887 delivers a next voltage $0.08 \mathrm{~V}$. 
Fig. 6. Flexible Sensor with bent to $0^{\circ}$ (sparkfun.com)

Upon bending the flexible sensor voltage acquired by the PIC16F887 gradually increase until it reaches a position $90^{\circ}$ (Fig. 8) to record a voltage of approximately $4.8 \mathrm{~V}$.

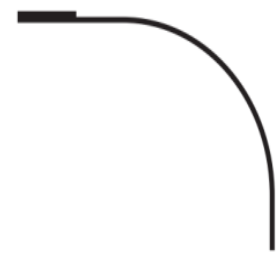

Fig. 7. Flexible Sensor with bent to $90^{\circ}$ (sparkfun.com)

The data sent to the microcontroller are analog signals, therefore requires the use of the PIC16F887, which has 13 ports for ADC (Analog to Digital Converter). The program employs the digitized signal delivered by the conditioning circuit. This data is compared to a record of variations of each of the sensors, to identify the letters.

To identify the alphabet letter we perform a simultaneous reading of all sensors and the algorithm in the microcontroller identifies each letter, based on the record of voltage presented for each sensor.

Another very important component for the identification of some alphabet letters as it can be seen in Fig. 8, is the accelerometer. It is used to identify the position of the hand in the 3 coordinate axes (Fig. 9), because certain letters are identified not only by the position of the fingers, but also by the movement produced by hand.

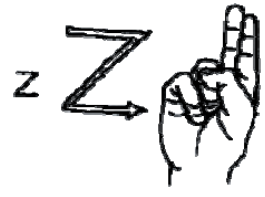

Fig. 8. Position of the fingers to the letter $\mathrm{Z}$ (scoutsanpedro.cl)

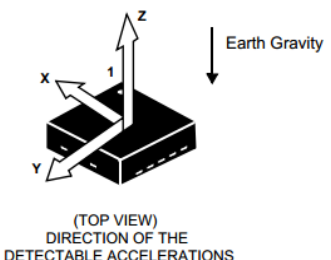

Fig. 9. Representation of the use of accelerometer MMA8452Q in 3 axes (freescale.com)

By using all of these components together can achieve the correct identification of all the letters of universal fingerspelling.

\section{B. Translation}

The components included in the translation stage are:

- Display LCD
- EMIC 2 Text to Speech Module \#30016 (parallax.com)

- Horn

This phase can be divided into two types of translations: visual and auditory.

The visual is based on post information acquired by the program to a LCD Display, with this the person can see if there was a correct translation or detection of each letter was successful. And so do the correct translation to audio through a voice synthesizer.

And the most important part of the research is the hearing translation, which is carried out with a device called "EMIC 2 Text to Speech Module". This component is based on the data reception of serial ports, usually used from a PC or an interactive processor for transmitting data. This is used as a portable translator of text to speech.

\section{RESULTS}

The prototype was made by placing the sensors on the way previously described (Fig. 10 and 11).

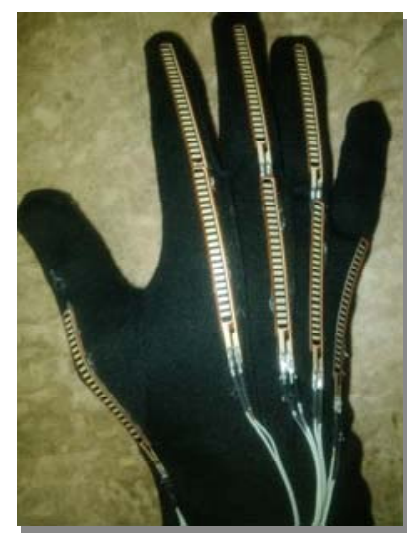

Fig. 10. Prototype (bend sensors)

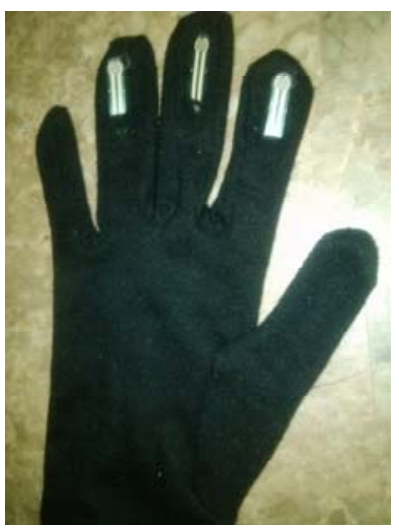

Fig. 11. Prototype (presure sensors)

A real example of the above may be the complete "HOLA" word in spanish (that means "HELLO" in english). Fig. 12 shows the signals of the sensors activated by each letter. 


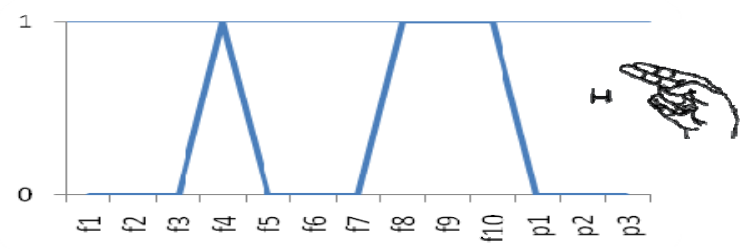

(a)

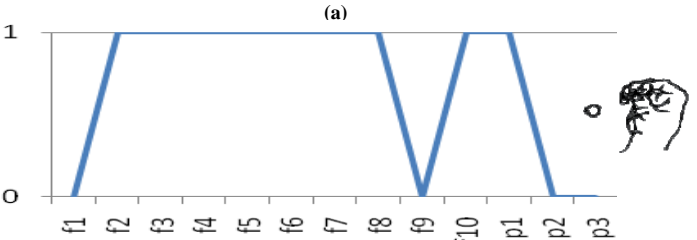

(b)
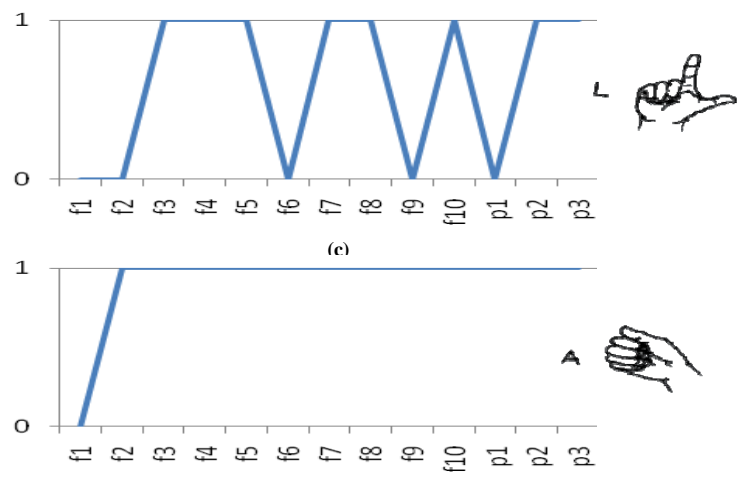

(d)

Fig. 12. Sensor activation signals (a) letter $\mathrm{H}$, (b) letter $\mathrm{O}$, (c) letter L y (d) letter A ( $\mathrm{f}=$ bend sensor , $\mathrm{p}=$ Force sensitive resistor)

\section{CONCLUSIONS}

We were able to make a prototype that translate the universal fingerspelling signals to audio signals, and with this make words.
The glove translator presented in this paper is designed to be a helpful tool for aid deaf-mute people in the everyday life communication.

Another important issue is the synthesizer voice, which will make the glove be a portable tool and easy to use in daily life.

The translator glove could be improved by assigning to the movement of a finger a greeting, phrase, or common questions and answers through an intelligent algorithm based on fuzzy logic so the person could communicate in a way even faster and he/she would not need to write letter by letter.

The authors are committed to upgrade the performance of the glove translator with materials that could make its cost accessible to all whom need it, in order to improve the quality of life of the deaf-mute community.

\section{REFERENCES}

[1] World Health Organization, Deafness and hearing loss, 2013, http://www.who.int/mediacentre/factsheets/fs300/en/

[2] Anbarasi Rajamohan, Hemavathy R., Dhanalakshmi M., “ DeafMute Communication Interpreter”, International Journal of Scientific Engineering and Technology, Volume 2 Issue 5, pp : 336-341, May 2013.

[3] N. Oess, J. Wanek and A. Curt, "Design and evaluation of a lowcost instrumented glove for hand function assessment," Journal of NeuroEngineering and Rehabilitation, vol. 9, pp. 1-11, January 2012.

[4] P. Espinoza Aguilar y H. Pogo León, Diseño y construcción de un guante prototipo electrónico de traducir el lenguaje de señas de una persona sordomuda al lenguaje de letras, Cuenca, ECU: Universidad Politécnica Salesiana, 2013, pp. 14-85.

[5] J. Leybón , M. Ramirez y V. Teboada , "Photo-Electric Sensor Applied to Hand Fingers Movement”, Computación y Sistemas, v.10 , pp. 1-11, 2006. 